\title{
The Drosophila Postsynaptic DEG/ENaC Channel ppk29 Contributes to Excitatory Neurotransmission
}

\author{
(1DAlexis Hill, ${ }^{1 \star}$ Xingguo Zheng, ${ }^{1,2 *}$ Xiling Li, ${ }^{3}{ }^{\circledR}$ Ross McKinney, ${ }^{1}{ }^{\oplus D}$ Dion Dickman, ${ }^{3}$ and Yehuda Ben-Shahar ${ }^{1}$ \\ ${ }^{1}$ Department of Biology, Washington University in St. Louis, St. Louis, Missouri 63130, ${ }^{2}$ Donald Danforth Plant Science Center, St. Louis, Missouri 63132, \\ and ${ }^{3}$ Department of Neurobiology, University of Southern California, Los Angeles, California 90089
}

The protein family of degenerin/epithelial sodium channels (DEG/ENaCs) is composed of diverse animal-specific, non-voltage-gated ion channels that play important roles in regulating cationic gradients across epithelial barriers. Some family members are also enriched in neural tissues in both vertebrates and invertebrates. However, the specific neurophysiological functions of most DEG/ENaC-encoding genes remain poorly understood. The fruit fly Drosophila melanogaster is an excellent model for deciphering the functions of DEG/ENaC genes because its genome encodes an exceptionally large number of DEG/ENaC subunits termed pickpocket (ppk) 1-31. Here we demonstrate that $p p k 29$ contributes specifically to the postsynaptic modulation of excitatory synaptic transmission at the larval neuromuscular junction. Electrophysiological data indicate that the function of $p p k 29$ in muscle is necessary for normal postsynaptic responsivity to neurotransmitter release and for normal coordinated larval movement. The $p p k 29$ mutation does not affect gross synaptic morphology and ultrastructure, which indicates that the observed phenotypes are likely due to defects in glutamate receptor function. Together, our data indicate that $\mathrm{DEG} / \mathrm{ENaC}$ ion channels play a fundamental role in the postsynaptic regulation of excitatory neurotransmission.

Key words: DEG/ENaC; Drosophila melanogaster; fruit fly; NMJ; synapse

Significance Statement

Members of the degenerin/epithelial sodium channel $(\mathrm{DEG} / \mathrm{ENaC})$ family are broadly expressed in epithelial and neuronal tissues. To date, the neurophysiological functions of most family members remain unknown. Here, by using the power of Drosophila genetics in combination with electrophysiological and behavioral approaches, we demonstrate that the DEG/ENaC-encoding gene pickpocket 29 contributes to baseline neurotransmission, possibly via the modulation of postsynaptic glutamate receptor functionality.

\section{Introduction}

The identities and functions of genes that regulate neuronal synaptic functions in health and disease remains a major goal of neuroscience research. Although the principle molecular mechanisms that regu-

Received Dec. 17, 2016; revised Jan. 30, 2017; accepted Feb. 12, 2017.

Author contributions: A.H., X.Z., D.D., and Y.B.-S. designed research;A.H., X.Z.,X.L., and R.M. performed research; A.H. contributed unpublished reagents/analytic tools; A.H., X.Z., X.L., R.M., and D.D. analyzed data; A.H., X.Z., D.D., and Y.B.-S. wrote the paper.

This work was supported by W.M. Keck and McDonnell Center Postdoctoral Fellowships to A.H.; National Institutes of Health (NIH) Grant NS-019546 and research fellowships from the Alfred P. Sloan, Ellison Medical, the Whitehall, Klingenstein-Simons, and Mallinckrodt Foundations to D.D.; and grants from the Klingenstein Foundation and the McDonnell Center for Cellular and Molecular Neurobiology, and NIH Grant 5R21-NS-089834 to Y.B.-S. We thank Aaron DiAntonio for antibodies; and the Bloomington Drosophila Stock Center at Indiana University and the fly community for fly strains. Anti-BRP and anti-GluRIIA antibodies, developed by E. Buchner and C. Goodman, respectively, were from the Developmental Studies Hybridoma Bank at the University of lowa, Department of Biology. We also thank Matthew Joens and James Fitzpatrick from the Washington University in St. Louis Center for Cellular Imaging, which is supported by the Washington University in St. Louis School of Medicine, The Children's Discovery Institute of Washington University in St. Louis and St. Louis Children's Hospital, the Foundation for BarnesJewish Hospital, the National Institute for Neurological Disorders and Stroke (Grant NS-086741), the Office of the NIH Director (Grant OD-021694), and the National Science Foundation (Grant 1633971).

${ }^{*}$ A.H. and X.Z. contributed equally to this research.

The authors declare no competing financial interests. late synapse formation and function are relatively well understood, mechanisms for synaptic plasticity, especially at the physiological timescale, are still mostly unknown. Here we describe a novel role for pickpocket ( $p$ pk) 29, a member of the degenerin/epithelial sodium channel (DEG/ENaC) family of non-voltage-gated sodium channels (Zelle et al., 2013), in the postsynaptic modulation of baseline excitatory neurotransmission at the Drosophila larval neuromuscular junction (NMJ).

Members of the DEG/ENaC family are exclusively found in animal genomes. They function as trimeric cation channels, which are expressed in both neuronal and non-neuronal tissues (Kellenberger and Schild, 2002; Jasti et al., 2007; Gonzales et al., 2009; Chen et al., 2015). DEG/ENaC channels can be gated by diverse extracellular stimuli, including extracellular ligands and

Correspondence should be addressed to Dr. Yehuda Ben-Shahar, Department of Biology, Washington University in St. Louis, St. Louis, M0 63130. E-mail: benshahary@wustl.edu.

DOI:10.1523/JNEUROSCI.3850-16.2017

Copyright $@ 2017$ the authors $\quad 0270-6474 / 17 / 373171-10 \$ 15.00 / 0$ 
mechanical forces (Ben-Shahar, 2011; Eastwood and Goodman, 2012).

Several studies in invertebrate and mammalian species suggest that some DEG/ENaC family members also directly contribute to synaptic functions (Younger et al., 2013; Urbano et al., 2014; Ievglevskyi et al., 2016; Miller-Fleming et al., 2016), which may explain their reported contributions to long-term potentiation, learning and memory (Wemmie et al., 2002), and addiction (Kreple et al., 2014). In addition, mutations in DEG/ENaCencoding genes have been implicated in neuropathologies such as multiple sclerosis and epilepsy (Wemmie et al., 2013). However, whether the observed neuronal and behavioral phenotypes of mutations in DEG/ENaC-encoding genes are due to presynaptic or postsynaptic processes is not well understood.

In contrast to mammalian genomes, which typically harbor eight to nine independent DEG/ENaC-encoding genes, the genome of the fruit fly Drosophila melanogaster encodes $>30$ independent family members, named ppk genes (Zelle et al., 2013). Analyses of mutations in several $p p k$ genes indicates that Drosophila DEG/ENaC channels contribute to diverse sensory functions such as salt taste (Liu et al., 2003), water sensing (Cameron et al., 2010; Chen et al., 2010), and the detection of mating pheromones (Lin et al., 2005; Liu et al., 2012; Lu et al., 2012; Starostina et al., 2012; Thistle et al., 2012). In addition, some $p p k$ genes have been implicated in the maintenance of synaptic homeostasis (Younger et al., 2013). Nevertheless, the specific molecular mechanisms by which these channels exert their synaptic functions remain elusive.

Here we report that $p p k 29$, which has been reported previously as a neuronally enriched Drosophila DEG/ENaC subunit implicated in pheromone-sensing functions (Thistle et al., 2012; Mast et al., 2014; Vijayan et al., 2014; Yuan et al., 2014), is also required for normal neurotransmission at the larval NMJ, a model glutamatergic synapse (Menon et al., 2013), via postsynaptic processes, possibly via modulation of postsynaptic glutamate receptors.

\section{Materials and Methods}

Fly stocks and genetics. Fruit flies (D. melanogaster) were raised on standard corn syrup-soy food (Archon Scientific) at $25^{\circ} \mathrm{C}$ and $60 \%$ relative humidity with a $12 \mathrm{~h}$ light/dark cycle. Unless specifically noted, the $w^{1118}$ strain was used as the "wild-type" (WT) control. All ppk29 and sei alleles described here were transgressed into the same wild-type background.

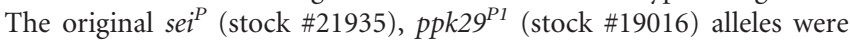
from the Bloomington Drosophila Stock Center at Indiana University (Bloomington, IN), and the $p p k 29^{P 2}$ allele was from The Exelixis Collection at the Harvard Medical School (stock \#f04205, Boston, MA). The elav (Lin and Goodman, 1994) and BG57 (Budnik et al., 1996) GAL4 lines were from the Bloomington Drosophila Stock Center at Indiana University. The transgenic lines UAS-ppk29 and UAS-seis3'UTR were described previously (Zheng et al., 2014).

Electrophysiology. Intracellular recordings were performed as described previously (Dickman et al., 2012). In brief, third-instar larvae were dissected in HL3 buffer ( $70 \mathrm{~mm} \mathrm{NaCl}, 5 \mathrm{~mm} \mathrm{KCl}, 10 \mathrm{~mm} \mathrm{MgCl}, 10 \mathrm{~mm} \mathrm{NaHCO}$, 5 $\mathrm{mm}$ trehalose, $5 \mathrm{~mm}$ HEPES, and $0.3 \mathrm{mM} \mathrm{Ca}^{2+}, \mathrm{pH} 7.2$ ). Intracellular recordings were performed from muscle 6 of segments A2 or A3. The average evoked junction potential (EJP) amplitude was calculated from the responses of 20 presynaptic stimuli (stimulus frequency, $0.2 \mathrm{HZ}$; stimulus duration, $3 \mathrm{~ms}$ ). Recordings were collected from muscles with an initial membrane potential between -60 and $-80 \mathrm{mV}$ by using intracellular electrodes with resistances of 7-20 M $\Omega$, filled with $3 \mathrm{M} \mathrm{KCl}$ and input resistances $\geq 4 \mathrm{M} \Omega$. No differences in passive membrane properties of the muscle were observed, including resting potential, input resistance, and capacitative transients (Table 1; data not shown). Effects of $p p k 29$ mutation on the expression of synaptic homeostasis were studied using current-clamp recordings. Phil-
Table 1. Muscle input resistance and resting membrane potential are reported for the electrophysiological recordings presented in Figures 1 and 2

\begin{tabular}{|c|c|c|c|}
\hline & Group & Input resistance ( $M \Omega$ ) & $\begin{array}{l}\text { Resting membrane } \\
\text { potential (mV) }\end{array}$ \\
\hline \multirow[t]{4}{*}{ Figure 1} & WT & $11.75 \pm 1.36$ & $64.41 \pm 1.22$ \\
\hline & WT + PhTX & $11.44 \pm 1.21$ & $64.59 \pm 1.79$ \\
\hline & $p p k 2 g^{p 1}$ & $9 \pm 1.24$ & $65.42 \pm 1.52$ \\
\hline & $p p k 29^{p 1}+P h T X$ & $8.67 \pm 1.14$ & $66.07 \pm 2.42$ \\
\hline$p$ value $^{a}$ & & 0.2532 & 0.8904 \\
\hline \multirow[t]{4}{*}{ Figure 2} & WT & $10.75 \pm 1.21$ & $66.47 \pm 2.29$ \\
\hline & $p p k 29^{p 1}$ & $8.46 \pm 1.00$ & $67.68 \pm 2.28$ \\
\hline & $B G 57>p p k 29 ; p p k 29^{P 1}$ & $8.47 \pm 0.81$ & $66.08 \pm 0.94$ \\
\hline & elav $>p p k 29 ; p p k 29^{P 1}$ & $10.47 \pm 0.56$ & $68.49 \pm 1.19$ \\
\hline$p$ value $^{a}$ & & 0.1136 & 0.6138 \\
\hline
\end{tabular}

Data are the mean \pm SEM. No significant differences were observed. $n=8-19$ per group.

${ }^{a}$ Determined by ANOVA.

anthotoxin (PhTX; Sigma-Aldrich) was dissolved in distilled $\mathrm{H}_{2} \mathrm{O}$, diluted to $10 \mu \mathrm{M}$ in HL3 buffer with $0.4 \mathrm{mM} \mathrm{Ca}^{2+}$. Spontaneous miniature EJPs (mEJPs) and EJPs were recorded, and the quantal content for each recording was estimated by dividing the mean EJP amplitude by the mean $\mathrm{mEJP}$ amplitude. The homeostatic response was determined by comparing the amplitude of spontaneous and evoked potentials before and after the application of PhTX. For the two-electrode voltage-clamp (TEVC) recordings, muscles were clamped at $-70 \mathrm{mV}$. All signals were recorded at a sampling rate of $10 \mathrm{kHz}$ and low-pass filtered at $1 \mathrm{kHz}$. Recordings were analyzed by using Clampfit 10.5 (Molecular Devices), and MiniAnalysis (Synaptosoft).

Larval locomotion velocity assay. Third-instar larvae were individually placed onto an agar plate $(60 \mathrm{~mm}$ dish, $3 \% \mathrm{w} / \mathrm{v})$ and allowed to roam freely for $5 \mathrm{~min}$. Locomotion was video recorded at 5 frames/s (Logitech C920 Webcam), and the centroid position of each larva was determined using custom video analysis software (R. McKinney and Y. Ben-Shahar, unpublished software). Velocities were calculated for every frame, based on the distance traveled since the previous frame, and the mean velocity for each larva across all frames was used for comparison.

Larval rollover assay. Third-instar larvae were individually placed onto an agar plate $(60 \mathrm{~mm}$ dish, $3 \% \mathrm{w} / \mathrm{v})$ and allowed to acclimate to the behavioral arena for $2 \mathrm{~min}$. Subsequently, the larva was gently rolled onto its dorsum with a soft paint brush and briefly $(1 \mathrm{~s})$ held in position before being allowed to roll back to an upright body position. Each larva was tested three consecutive times with $10 \mathrm{~s}$ resting intervals. The average time for the three trials was recorded as the rollover time for each larva.

Immunohistochemistry. Third-instar larvae were dissected in ice-cold Schneider's Insect Medium (Sigma-Aldrich) and fixed in Bouin's fixative for 10 min while pinned on a SYLGARD plate. Larvae were then washed in PBS plus $0.1 \%$ Triton-X (PBST) and blocked for $30 \mathrm{~min}$ in PBST plus $5 \%$ goat serum at room temperature. Larvae were incubated in primary antibody overnight at $4^{\circ} \mathrm{C}$. Secondary antibodies were applied for $2 \mathrm{~h}$ at room temperature. The following primary and secondary antibodies (dilution, source) were used: mouse anti-BRP (Bruchpilot; 1:500; catalog \#nc82, Developmental Studies Hybridoma Bank at the University of Iowa, Iowa City, IA; RRID:AB_528108); mouse anti-GluRIIA (1:100; catalog \#8B4D2 (MH2B), Developmental Studies Hybridoma Bank at the University of Iowa; RRID:AB_528269); rabbit anti-GluRIIB (1:2000; catalog \#GluRIIB; RRID:AB_2568753; Marrus et al., 2004); rabbit antiGluRIIC (1:2500; catalog \#GluRIIC; RRID:AB_2568754; Marrus et al., 2004); Alexa Fluor 568-conjugated goat anti-rabbit (1:500; catalog \#A11011, ThermoFisher Scientific; RRID:AB_143157); and Alexa Fluor 647-conjugated goat anti-mouse (1:500; catalog \#A-21240, ThermoFisher Scientific; RRID:AB_2535809). FITC-conjugated goat anti-HRP (1:1000; catalog \#123-095-021, Jackson ImmunoResearch; RRID: AB_2314647) was used during the secondary antibody incubation step to label neuronal processes. After staining, larval preparations were equilibrated in 70\% glycerol/PBS and mounted with VectaShield (Vector Laboratories). Larval preparations were imaged using an A1Si laser-scanning confocal microscope (Nikon). Nikon Elements Software was used to capture, process, and analyze images. To quantify immunofluorescence 
intensity levels, $z$-stacks through the entirety of the NMJ were rendered as maximum projection images and analyzed in Image (Schindelin et al., 2012), while blind to genotype. Mean background fluorescence intensity was subtracted from the mean intensity at the synapse and then normalized relative to wild type.

Electron microscopy. Third-instar larvae were dissected in PBS, and fixed in $2.5 \%$ glutaraldehyde/2\% paraformaldehyde in PBS overnight at $4^{\circ} \mathrm{C}$. Samples were then rinsed in PBS and subjected to secondary fixation for $1 \mathrm{~h}$ on ice in $1 \%$ osmium tetroxide $/ 1.5 \%$ potassium ferrocyanide in PBS. Next, samples were washed in ultrapure water and then en bloc stained for $1 \mathrm{~h}$ with $2 \%$ aqueous uranyl acetate. Samples were then dehydrated in a graded acetone series $(50 \%, 70 \%, 90 \%, 100 \% 2 \times)$, infiltrated with microwave assistance (BioWave Pro, Pelco) into LX112 resin, and embedded in aluminum weighing dishes. Samples were cured in an oven at $60^{\circ} \mathrm{C}$ for $48 \mathrm{~h}$. A small region was excised and glued onto a blank stub oriented at a $45^{\circ}$ angle. Seventy nanometer sections were cut, counterstained with uranyl acetate and lead citrate, and imaged on a transmission electron microscope (model JEM-1400, JEOL) at $80 \mathrm{KeV}$. Analysis of synaptic vesicle size was conducted on seven boutons per genotype, using sections containing at least one active zone, comprising a total of 300 synaptic vesicles per genotype. All vesicle analysis was conducted using ImageJ while blind to genotype.

Real-time $q R T-P C R$. Total RNA was extracted from pools of 10 thirdinstar larvae (5 males and 5 females) using TRIzol reagent (ThermoFisher Scientific). First-strand cDNA was prepared using SuperScript II reverse transcriptase (ThermoFisher Scientific). Gene-specific assays were conducted using iTaq Universal SYBR Green 2X Supermix (Bio-Rad) on a CFX Connect Real-Time PCR Detection System (Bio-Rad). Each RNA sample was run in triplicate. The housekeeping gene $r p 49$ was used as a loading control. Relative expression data were analyzed and presented as fold differences by using the $\Delta \Delta C T$ method, as previously described (Lu et al., 2012). The following primer sequences were used: $r p 49$-forward, $5^{\prime}$-CACC AAGCACTTCATCCG-3'; rp49-reverse, 5'-TCGATCCGTAACCGA TGT-3'; GluRIIA-forward, 5' -TTCAATCCCTCGGCCTTCAC-3'; Glu RIIA-reverse, 5'-GTCCGGTAATCAGAGCCCAG-3'; GluRIIB-forward, 5'-CGTCGACGACAGCATTGTTC-3'; GluRIIB-reverse, 5'-CGACGA TGTCGCTGTTTGTC-3'; GluRIIC-forward, 5'-TCATGAAAGGGAC TTCCGGC-3'; and GluRIIC-reverse, 5'-ACGTTATCCGCTGTCCATCC- $3^{\prime}$.

Statistical analyses. Quantitative neurophysiological, immunostaining, and behavioral data were analyzed using StatView (Scientific Computing) and Prism7 (GraphPad). Data were analyzed using either Student's $t$ test or one-way ANOVA followed by a Tukey's post hoc analysis $(p<0.05)$ when pairwise comparisons were appropriate. Error bars denote the SEM.

\section{Results}

$p p k 29$ contributes to spontaneous neurotransmitter release in excitatory synapses

In previous work, we demonstrated that the $p p k 29$ gene regulates the neuronal response to heat stress via protein-independent, post-transcriptional regulation of the voltage-gated potassium channel seizure (sei; Zheng et al., 2014). However, the contribution of the PPK29 protein to neuronal physiology remained unknown. Several studies by others suggested that $p p k 29$ contributes to pheromone sensing via an unknown mechanism (Thistle et al., 2012; Mast et al., 2014; Vijayan et al., 2014; Yuan et al., 2014). However, examination of tissue expression data and brain in situ hybridization revealed that $p p k 29$ is also expressed in central neurons (Zheng et al., 2014). Independently, several studies suggested that specific $\mathrm{DEG} / \mathrm{ENaC}$-encoding genes contribute to synaptic functions in mammals and Drosophila (Wemmie et al., 2002; Voglis and Tavernarakis, 2008; Younger et al., 2013; Du et al., 2014; Urbano et al., 2014). Specifically, previous work indicated that the DEG/ENaC-encoding genes $p p k 11$ and $p p k 16$ regulate the presynaptic homeostatic response of Drosophila larval motor neurons after application of the glutamate receptor antag- onist PhTX (Frank et al., 2006; Younger et al., 2013), which is thought to be mediated via neuronal upregulation of neurotransmitter release quanta in response to the reduced availability of postsynaptic glutamate receptors (Davis, 2013).

Using the larval NMJ model for the glutamatergic synapse (Collins and DiAntonio, 2007; Menon et al., 2013), we found no effect of the $p p k 29$ mutation (transposon insertion line $p p k 29^{p I}$ ) on PhTX-induced synaptic homeostasis (Fig. 1). Following application of PhTX, spontaneous mEJP amplitudes were reduced, as expected, and there was a concomitant increase in quantal content to compensate, in both wild-type and ppk29 mutants (Fig. 1). However, we observed a difference between controls and ppk29 mutants on baseline spontaneous neurotransmission. Specifically, we found that, compared with wild-type animals, larvae with the $p p k 29$ mutation exhibited decreased mEJP amplitude $\left(n=8-16\right.$ per group; ANOVA: $F_{(3,38)}=28.624, p<0.0001$; Tukey's post hoc test: $p=0.001$ ) and that PhTX application further decreases mEJP amplitude in both genotypes (Tukey's post hoc test: WT baseline vs PhTX, $p<0.0001 ; p p k 29^{P 1}$ baseline vs PhTX, $p=0.019$; Fig. $1 A, B$ ). In contrast, we did not observe effects of genotype or PhTX application on stimulus-evoked EJPs (ANOVA: $F_{(3,38)}=2.091, p=0.1176$; Fig. $1 C$ ). These data show that the basal NMJ synaptic quantal content is increased in $p p k 29$ mutants relative to WT controls (ANOVA: $F_{(3,38)}=26.700, p<$ 0.0001; Tukey's post hoc test: $p=0.0336$ ) without measurable differences in PhTX-driven synaptic homeostasis (Tukey's post hoc test: WT baseline vs PhTX, $p<0.0001 ; p p k 29^{p 1}$ baseline vs PhTX, $p=0.0021$; Fig. $1 D$ ). Based on these data, we hypothesized that the $\mathrm{DEG} / \mathrm{ENaC}$-encoding gene $p p k 29$ plays a previously unrecognized synaptic role by regulating the amplitude of spontaneous neurotransmission.

\section{$p p k 29$ contributes to the regulation of synaptic transmission via postsynaptic processes}

Previous studies suggested that the activity of specific DEG/ENaC channels affects synaptic transmission at either presynaptic or postsynaptic sites (Wemmie et al., 2002; Younger et al., 2013; Du et al., 2014; Urbano et al., 2014). We reasoned that the observed impact of the ppk29 mutation on mini-amplitude could be due to either presynaptic processes, via altered structural morphology or the size of synaptic vesicles, or postsynaptic processes via altered glutamate receptor functions. Therefore, we next investigated whether the contribution of ppk29 to synaptic transmission is mediated via presynaptic or postsynaptic processes by using the UAS-GAL4 binary expression system (Brand and Perrimon, $1993)$ to rescue the $p p k 29$ mutation in a tissue-specific manner. Our data show that the postsynaptic rescue of the $p p k 29$ mutation with the muscle-specific BG57-GAL4 driver (Budnik et al., 1996) is sufficient to restore WT levels of the mEJP amplitude ( $n=$ 8-19; ANOVA: $F_{(3,55)}=11.744, p<0.0001$; Tukey's post hoc test: WT vs $p p k 29^{P 1}, p=0.0043 ; p p k 29^{P 1}$ vs BG57>UAS-ppk29; $p p k 29^{p 1}, p<0.0001$; Fig. 2A). In contrast, rescue of the $p p k 29$ mutation by expressing the $p p k 29 \mathrm{cDNA}$ specifically in neurons with the pan-neuronal elav-GAL4 driver (Lin and Goodman, 1994) does not rescue the phenotype (Tukey's post hoc test: $p p k 29^{P 1}$ vs elav $>U A S-p p k 29 ; p p k 29^{P 1}, p=0.4204$; Fig. $\left.2 A\right)$.

Surprisingly, we observed a decrease in mEJP frequencies in the neuronal rescue animals when compared with $p p k 29$ mutant animals $\left(n=8-19\right.$; ANOVA: $F_{(3,55)}=3.818, p=0.0148$; Tukey's post hoc test: $p p k 29^{p 1}$ vs elav $>U A S-p p k 29 ; p p k 29^{P 1}, p=0.0147$; Fig. 2B). However, we did not observe effects of the ppk29 mutation on mEJP frequencies relative to wild-type animals (Tukey's post hoc test: WT vs $\left.p p k 29^{P 1}, p=0.9807\right)$. Therefore, we conclude 
A
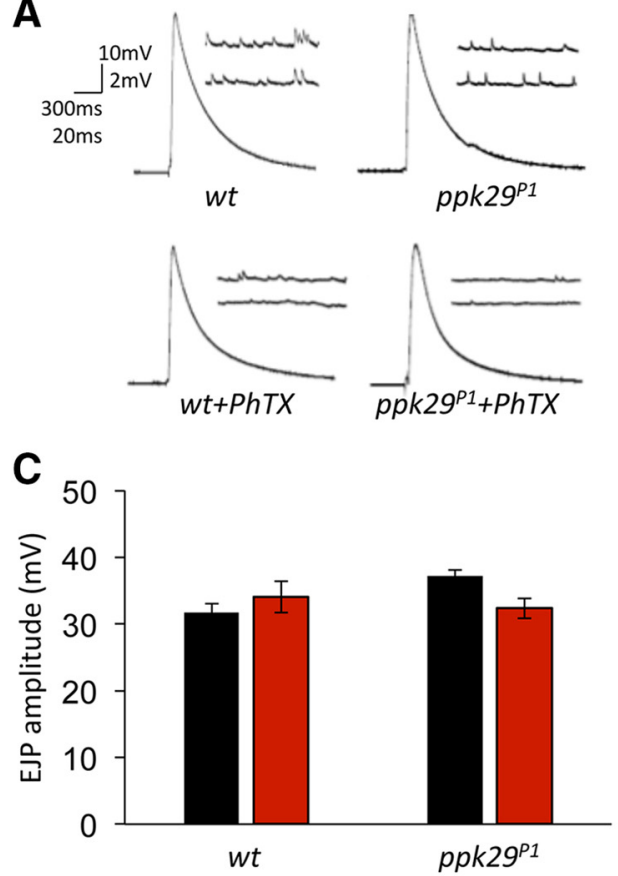

B
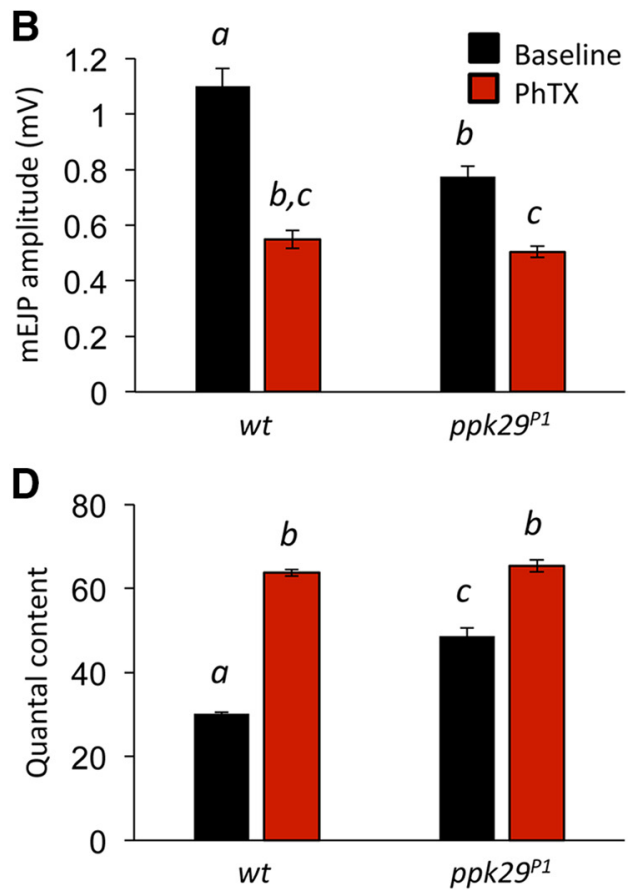

Figure 1. ppk29 mutants display normal homeostatic synaptic plasticity yet decreased response to spontaneous release at the larval NMJ. $\boldsymbol{A}$, Representative traces of mEJPs and EJPs before and after treatment with the glutamate receptor antagonist PhTX. $\boldsymbol{B}$, Compared with wild-type controls, ppk29 mutants display decreased mEJP amplitude. Both wild-type and ppk29 mutants display decreased mEJP amplitude following PhTX treatment. C, There is no effect of genotype or PhTX treatment on amplitude of evoked EJPs. $\boldsymbol{D}$, ppk29 mutants display increased quantal content compared with wild-type controls, and both genotypes display increased quantal content by PhTX treatment compared with baseline. Data are presented as the average \pm SEM. $n=8-16$ recordings/group for all experiments. Letters above bars represent statistical significance, $p<0.05$ (one-way ANOVA followed by Tukey's post hoc test).

that the neuronal impact of ppk29 overexpression on mEJP frequency is likely a gain-of-function phenotype. We also did not observe effects of the ppk29 mutation or expression of the ppk29 cDNA in muscle or neurons on EJP amplitude $(n=8-19$; ANOVA: $F_{(3,55)}=0.756, p=0.5235$; Fig. $2 C$ ). In contrast to Figure $1 D$, the overall effect of the $p p k 29$ mutation and rescue on synaptic quantal content in this experiment was just below statistical significance $\left(n=8-19\right.$; ANOVA: $F_{(3,55)}=2.762, p=0.0506$; Fig. 2D). Nevertheless, together, these data indicate that $p p k 29$ is required in the postsynaptic cell for the normal neurophysiological response to spontaneous neurotransmitter release at the NMJ.

Rollover behavior is abnormal in ppk29 mutant larvae

Since neurophysiological studies indicate that some baseline NMJ synaptic functions are abnormal in ppk29 mutant larvae, we next tested the hypothesis that $p p k 29$ is required for normal larval locomotion. To our surprise, we found that the overall crawling velocity of $p p k 29$ mutant larvae was not different from wild-type controls ( $n=8-9$ larvae/genotype; ANOVA: $F_{(2,23)}=1.364$; Fig. $\left.3 A\right)$.

Nevertheless, in contrast to simple forward locomotion, ppk29 mutants took significantly longer to right themselves after being rolled over onto their dorsal side $(n=12$ larvae/genotype; ANOVA: $F_{(2,33)}=12.807, p<0.001$; Tukey's post hoc test: WT vs $p p k 29^{P 1}, p<0.0001$; WT vs $p p k 29^{P 2}, p=0.0186$; Fig. $\left.3 B\right)$. Rescuing the ppk29 mutation selectively in muscles was sufficient to restore wild-type-like performance in this paradigm $(n=48$ larvae/genotype; ANOVA: $F_{(2,141)}=14.688, p<0.001$; Tukey's post hoc test: WT vs $p p k 29^{P 1}, p<0.0001 ; p p k 29^{P 1}$ vs BG57>UAS$p p k 29 ; p p k 29^{P 1}, p=0.007$; Fig. $\left.3 C\right)$. Expressing the $p p k 29$ cDNA in neurons did not rescue the mutant phenotype $(n=48$ larvae/ genotype; ANOVA: $F_{(2,141)}=8.329, p=0.0004$; Tukey's post hoc test: WT vs $p p k 29^{P 1}, p=0.0191 ; p p k 29^{P 1}$ vs elav>UAS-ppk29; $p p k 29^{P 1}, p=0.4229$; Fig. $3 D$ ). Together, these results demonstrate that $p p k 29$ expression in muscle is required for executing complex motor output sequences required for the larval rollover behavior, but not for simpler basal locomotion. In previous work, we demonstrated that ppk29 mRNA contributes to neuronal excitability and the organismal response to heat stress by acting as a regulatory natural antisense transcript of the potassium channel sei (Zheng et al., 2014). However, we did not observe any effects of the sei mutation on larval rolling behavior $(n=24$ larvae/ genotype; ANOVA: $F_{(1,46)}=1.573, p=0.2161$; Fig. $3 E$ ). Since our previous work has shown that mutations in $p p k 29$ affected neuronal physiology via the upregulation of sei expression levels (Zheng et al., 2014), we reasoned that if the postsynaptic effect of the ppk29 mutation on the larval rollover phenotype is due to upregulation of sei transcripts, then overexpressing sei in muscles should phenocopy the $p p k 29$ mutant phenotype. Instead, we observed an actual decrease in rollover time in larvae overexpressing sei in muscles $\left(n=24\right.$ larvae/genotype; ANOVA: $F_{(1,46)}=4.494$, $p=0.0394$; Fig. $3 F)$. Conversely, sei overexpression in neurons had no effect on larval behavior $(n=24$ larvae/genotype; ANOVA: $F_{(1,46)}=1.177, p=0.2836$; Fig. $\left.3 G\right)$. Together, these data indicate that the effects of the $p p k 29$ mutation on larval rollover behavior are likely independent of its mRNA-dependent interaction with sei in neurons.

\section{ppk29 is not required for synaptic development}

The neurophysiological and behavioral data presented above indicate that ppk29 mutant animals exhibit abnormal spontaneous excitatory neurotransmission at the NMJ. In past reports, similar phenotypes have been associated with abnormal synaptic development (Hanson and Landmesser, 2004; An et al., 2010; Kim et al., 2012; Choi et al., 2014). Consequently, we 
A

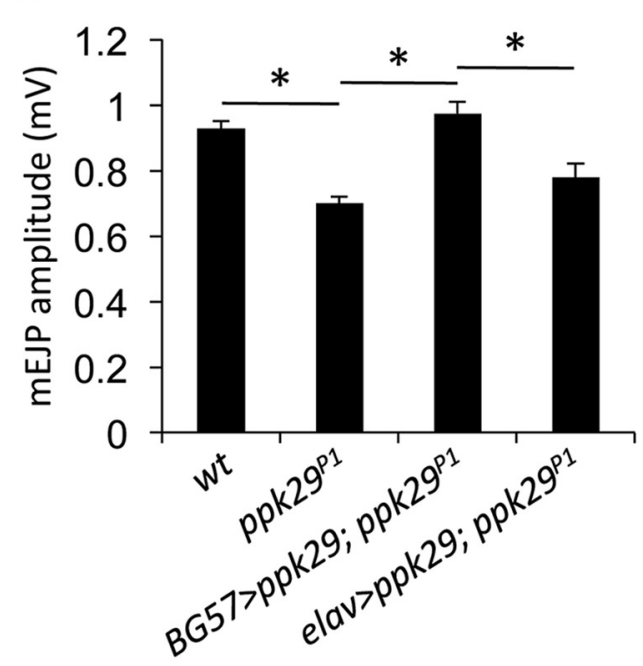

C

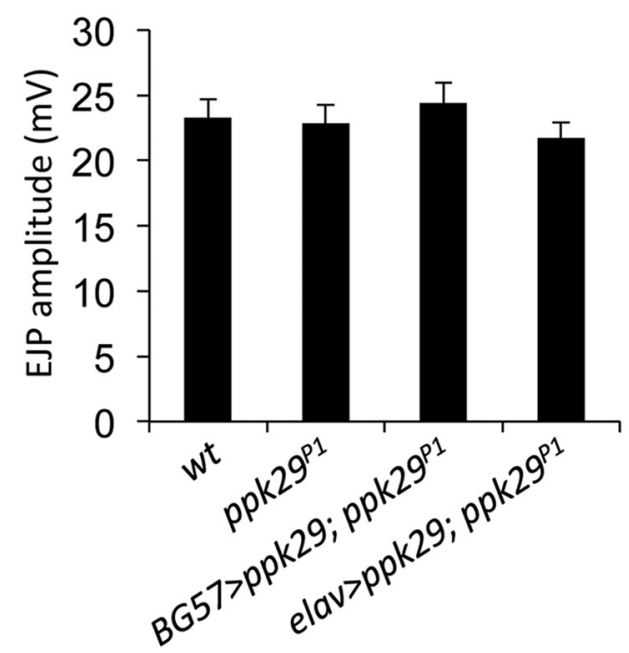

B

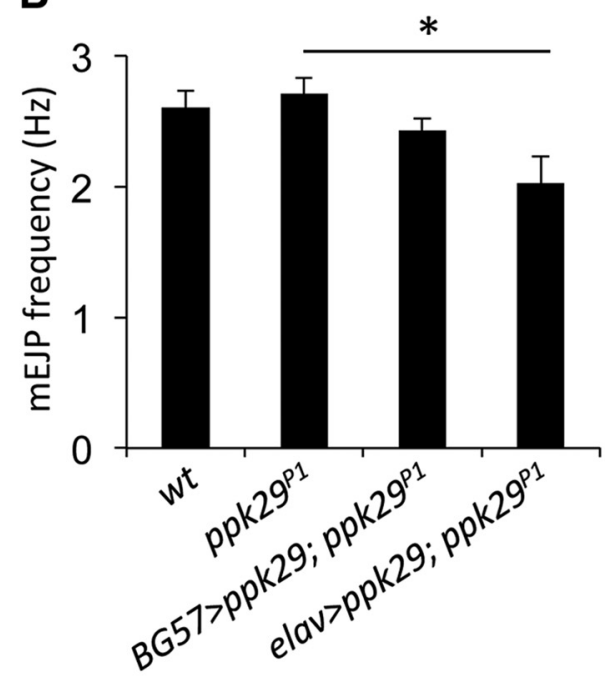

D

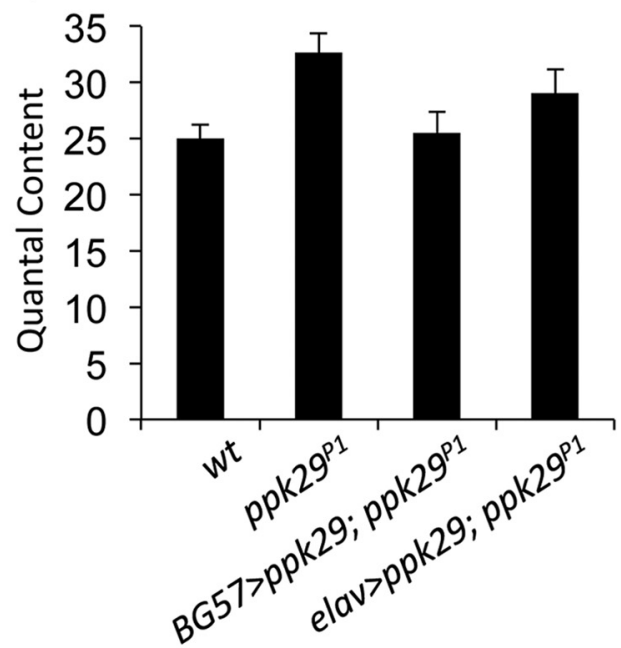

Figure 2. $\quad$ ppk29 plays a postsynaptic role in the response to spontaneous release. $\boldsymbol{A}$, The decreased mEJP amplitude observed in $p p k 29$ mutants is rescued by the expression of $p p k 29$ cDNA in muscles (using the BG57-Gal4 driver) but not in neurons (using the elav-Gal4 driver). $\boldsymbol{B}, p p k 29$ mutation has no impact on mEJP frequency, yet overexpression of $p p k 29$ cDNA in neurons (using the elav-Gal4 driver) decreases mEJP frequency. $\boldsymbol{C}$, There is no effect of $p p k 29$ mutation, rescue of $p p k 29$ expression in muscles, or rescue in neurons on EJP amplitude. $\boldsymbol{D}$, $p p k 29$ mutation and rescue in muscle or neurons has no significant effect on quantal content. Data are presented as the average \pm SEM. $n=8-19$ recordings/genotype for all experiments. ${ }^{*} p<0.05$ (one-way ANOVA followed by Tukey's post hoc tests).

used well established immunohistochemical markers for NMJ structures to test the hypothesis that the impact of the ppk29 mutation on spontaneous neurotransmission is a consequence of synaptic developmental structural abnormalities. However, in contrast to previous studies, we did not observe any effects of the ppk29 mutation on synaptic development or morphology as measured by the number of presynaptic boutons and the morphology of synaptic branching (boutons, $n=15-16 /$ group; ANOVA, $F_{(1,29)}=2.284, p=0.1415$; branches: $n=13-16 /$ group; ANOVA, $F_{(1,27)}=2.586, p=0.1195$; Fig. $\left.4 A-D\right)$. We also did not observe any differences in the number of active zones per NMJ labeled by the active zone marker protein $\operatorname{brp}(n=13-16 /$ group; ANOVA: $F_{(1,27)}=1.726, p=0.2000$; Fig. $4 E$ ) or in the apposition of presynaptic active zones with postsynaptic glutamate receptor clusters $\left(n=13-16\right.$ /group; ANOVA: $F_{(1,27)}=0.019, p=0.8914$; Fig. $4 F)$. Together, these data indicate that the abnormal spontaneous neurotransmission observed in ppk29 mutants is not likely to be a consequence of synaptic developmental or morphological abnormalities. ppk29 does not contribute to neurotransmitter vesicle size We next hypothesized that the observed decrease in mEJP amplitudes in ppk29 mutant animals (Figs. 1,2) may be due to changes in synaptic vesicle size. However, transmission electron microscopy images of NMJs revealed no effect of the mutation on the morphology of the active zone or the subsynaptic reticulum (Fig. $5 A-D)$. Furthermore, we observed no significant effects of the ppk29 mutation on the diameter of synaptic vesicles when analyzed by distribution histograms (Fig. $5 E$ ) or averages across boutons $\left(n=7\right.$ boutons/genotype; ANOVA: $F_{(1,12)}=0.583, p=$ 0.4597; Fig. $5 F$ ). We conclude that the ppk29 mutation does not impact synaptic vesicle size.

The ppk29 mutation is associated with altered glutamate receptor transcription and function

We did not observe any effects of the ppk29 mutation on the NMJ presynaptic morphology or synaptic vesicle size. Therefore, next we tested the hypothesis that postsynaptic ppk29 channels contribute to synaptic transmission via modulations of the function 

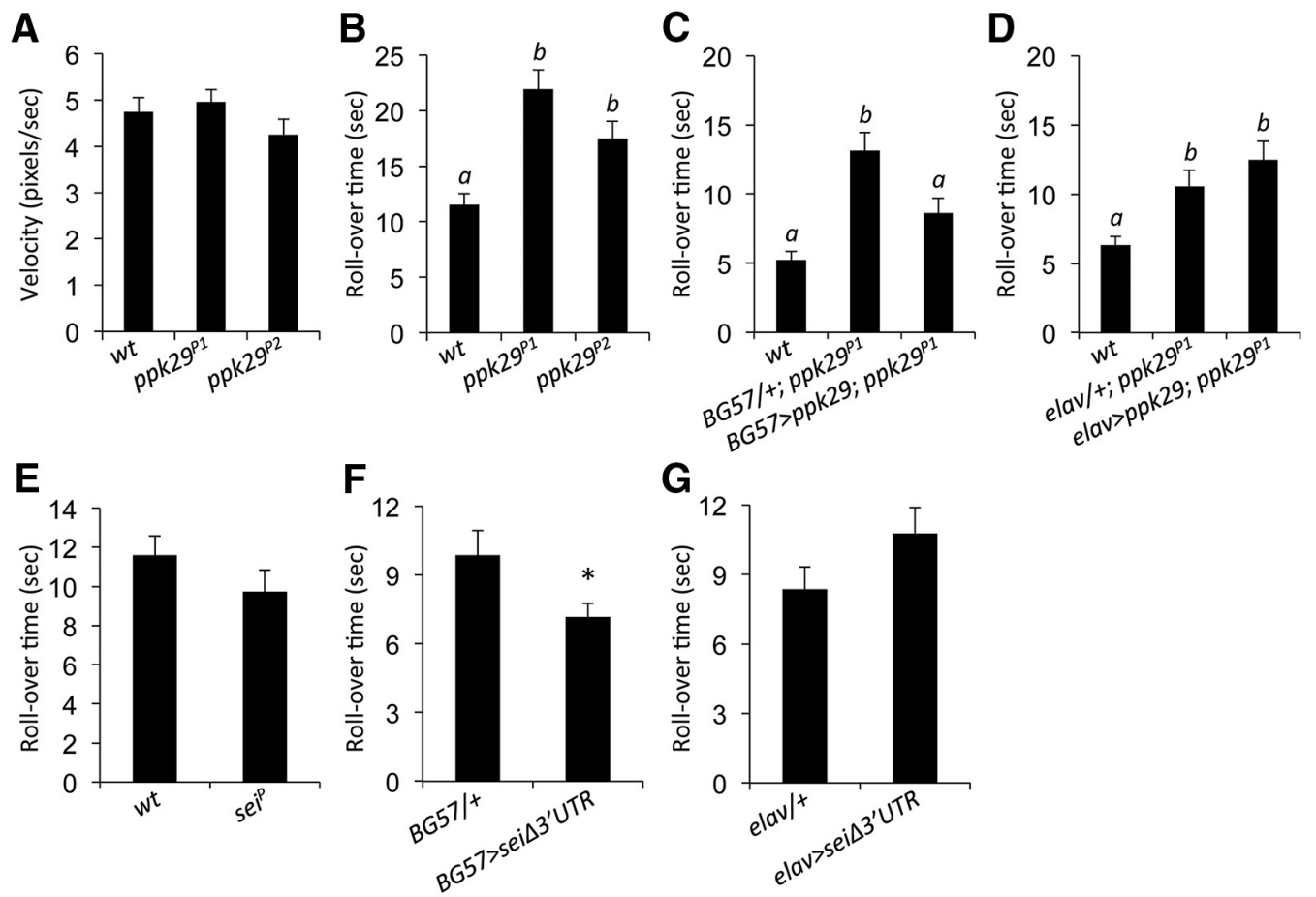

Figure 3. $p p k 29$ mutants display impaired larval roll behavior, which is rescued by muscle overexpression. $A$, There are no significant differences in average crawling velocity between wild-type controls and ppk29 mutants. $n=8-9$ larvae/genotype. B, ppk29 mutants display increased rollover time in a larval rollover assay. $n=12$ larvae/genotype. C, ppk29 mutant phenotype in larval rolling is rescued by the overexpression of $p$ pk29 CDNA in muscles (BG57-GAL4 promoter). $n=48$ larvae/genotype. $\boldsymbol{D}$, ppk29 mutant phenotype is not rescued by the overexpression of $p p k 29$ cDNA in neurons (elav-Gal4 promoter). $n=48$ larvae/genotype. $\boldsymbol{E}$, sei mutants display no significant difference in larval roll behavior compared with wild-type controls. $n=24$ larvae/genotype. $\boldsymbol{F}$, Overexpression of sei cDNA in muscles decreases rollover time. $n=24$ larvae/genotype. $G$, Overexpression of sei cDNA in neurons has no impact on larval roll behavior. $n=24$ larvae/genotype. Data are presented as the average \pm SEM. Letters above bars represent statistical significance, $p<0.05$ (one-way ANOVA followed by Tukey's post hoc tests); ${ }^{*} p<0.05$ (Student's $t$ test).
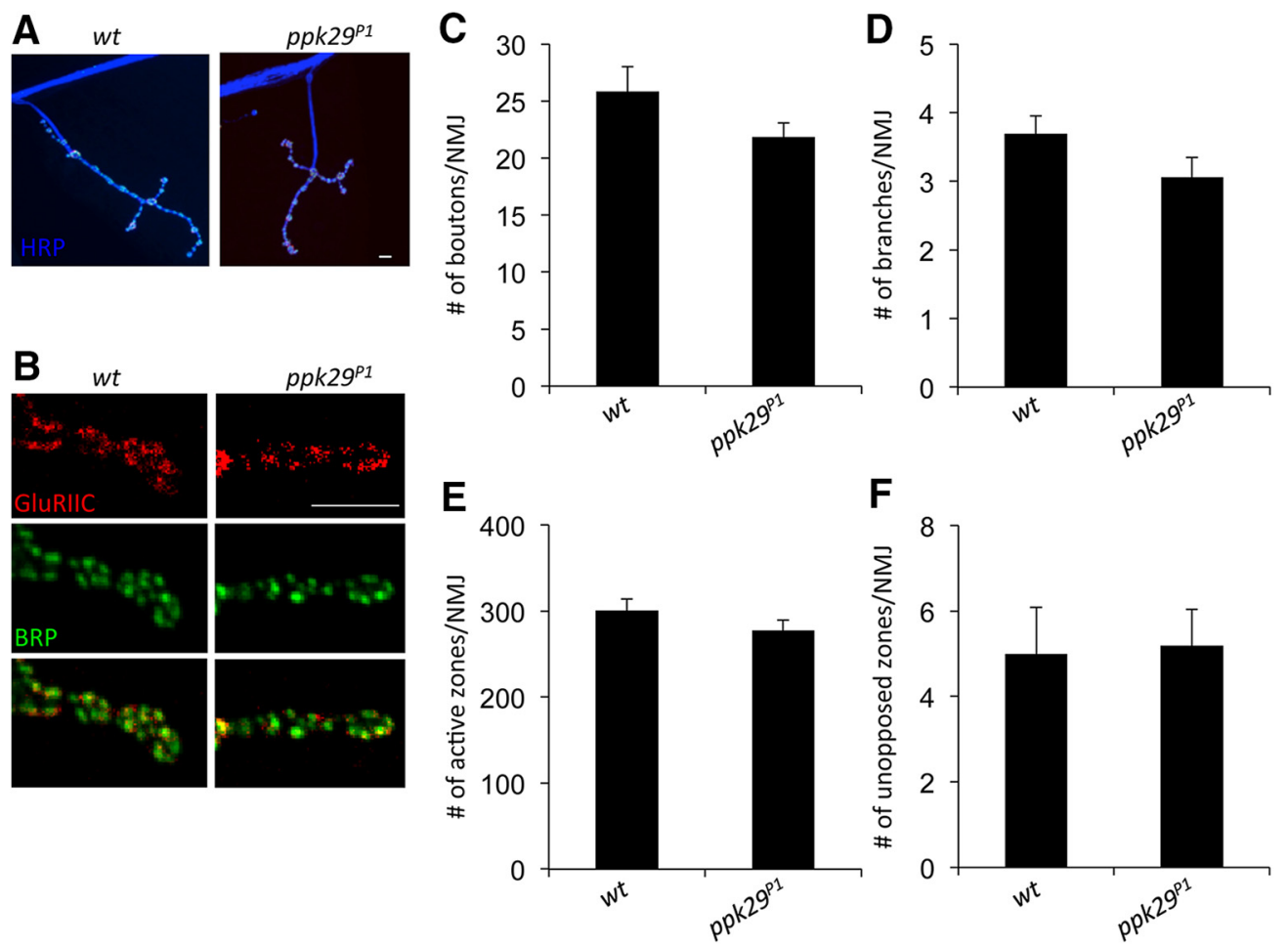

Figure 4. $\quad$ ppk29 mutants have normal presynaptic morphology and ultrastructure at the larval NMJ. $A, B$, Representative images of muscle 4 NMJs stained for HRP (blue), glutamate receptor subunit GluRIIC (red), and presynaptic active zone-associated protein BRP (green). C, D, ppk29 mutants display no significant difference in the number of presynaptic boutons or branches per NMJ, as assessed by HRP staining. $\boldsymbol{E}$, ppk29 mutants display no significant difference in the number of active zones per NMJ, as assessed by BRP puncta. $\boldsymbol{F}$, ppk29 mutants display no significant difference in the number of active zones unopposed by glutamate receptor clusters, as assessed by coimmunostaining for BRP and GluRIIC. Data analyzed using Student's $t$ test and presented as the average \pm SEM. $n=13-16 \mathrm{NMJs}$ analyzed/genotype. Scale bars, $5 \mu \mathrm{m}$. 

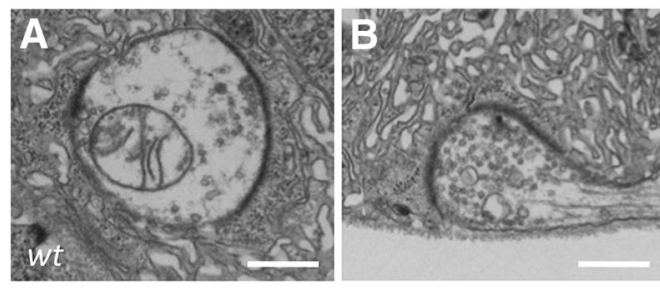

E

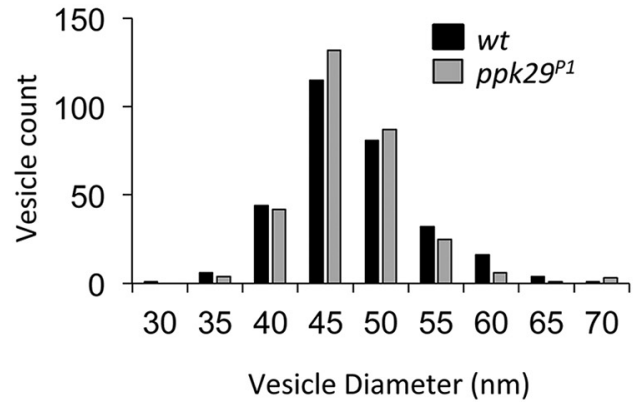

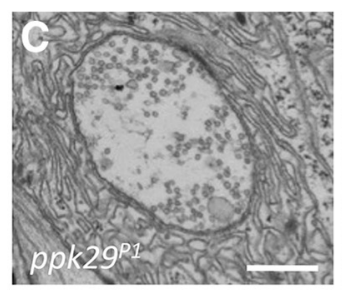

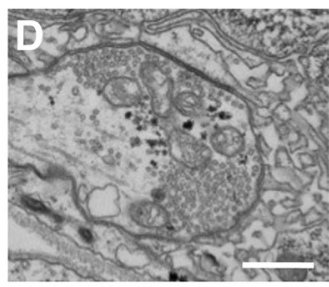

$\mathbf{F}$

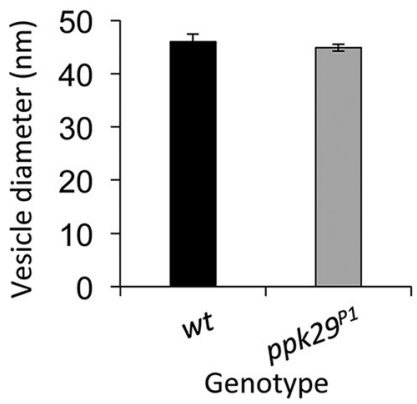

Figure 5. ppk29 mutants have normal ultrastructure and synaptic vesicle size. $\boldsymbol{A}-\boldsymbol{D}$, Representative EM images of the larval NMJ show similar synaptic ultrastructure in wild-type $(\boldsymbol{A}, \boldsymbol{B})$ and $p p k 29$ mutant $(\boldsymbol{C}, \boldsymbol{D})$ NMJs. $\boldsymbol{E}$, Histogram of all synaptic vesicles counted shows similar distributions between wild-type and ppk29 mutants. $\boldsymbol{F}$, Comparison of average vesicle diameter across genotypes shows similar average vesicle diameters. $n=7$ boutons per genotype. Data analyzed using Student's $t$ test and presented as average \pm SEM. Scale bar, $500 \mathrm{~nm}$.

$w t$
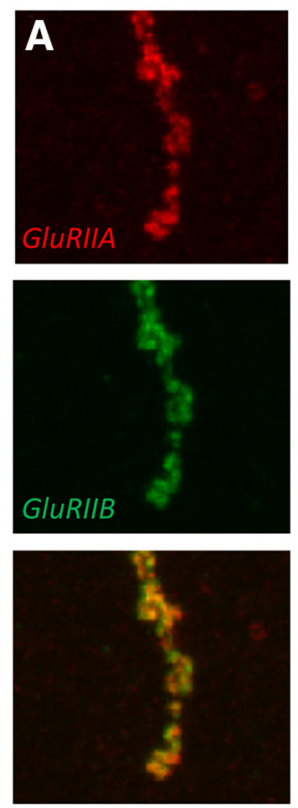

ppk29p1
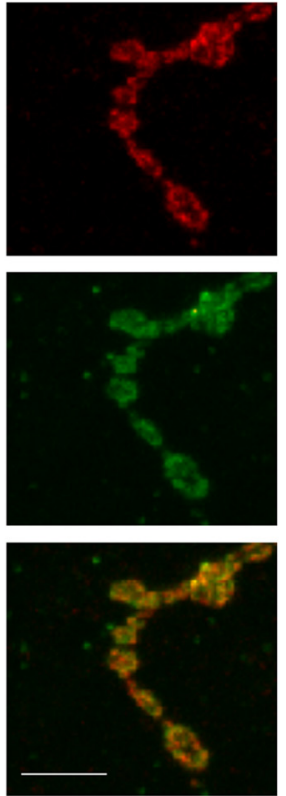

B

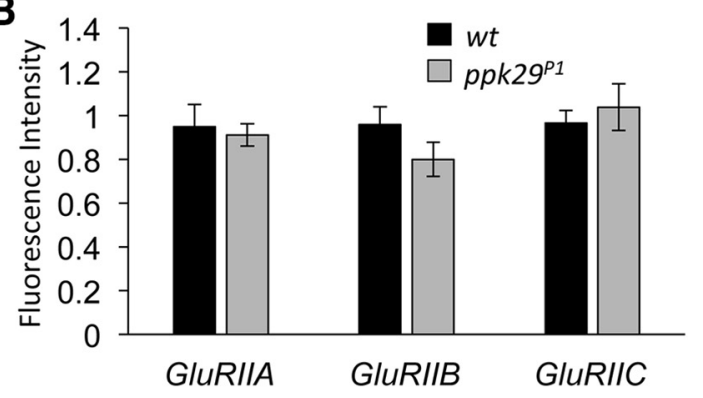

C

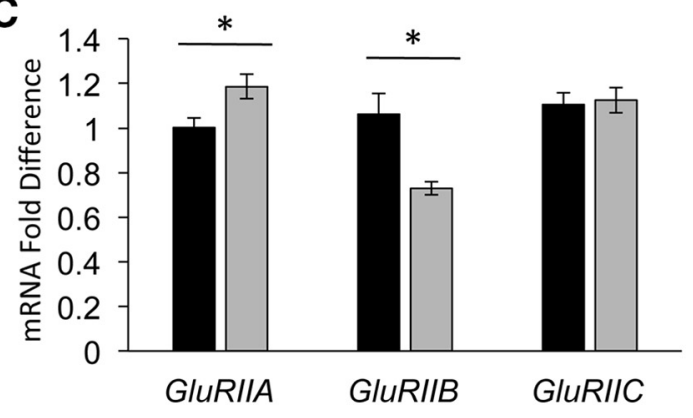

Figure 6. In ppk29 mutants, GIuRIIA and GluRIIB mRNA expression levels are altered but display normal localization to the NMJ. $A$, Images of muscle 4 NMJs show normal clustering of GluRIIIA (red) and GluRIIIB (green) subunits. B, Quantification of fluorescence intensity from immunostained NMJ $z$-stacks does not reveal statistically significant differences in levels of glutamate receptor subunits. $n=4-6$ images/genotype. C, qRT-PCR for glutamate receptor subunits shows that $p p k 29$ mutants have increased levels of GluRIIA and decreased levels of $G / u R I I B$, with no change in GluRIIC levels. $n=4$ samples/genotype. Data are presented as the average \pm SEM. ${ }^{*} p<0.05$ (Student's $t$ test). Scale bar, $5 \mu \mathrm{m}$.

of excitatory postsynaptic glutamate receptors. Previous studies have established that two postsynaptic ionotropic glutamate receptor subtypes are present at the Drosophila larval NMJ, which are defined by the association of either GluRIIA or GluRIIB subunits with the three common subunits GluRIIC, GluRIID, and GluRIIE. Previous studies showed that type A and type B receptors exhibit different rates of receptor desensitization in the presence of glutamate, with type A receptors carrying the bulk of the postsynaptic current (Petersen et al., 1997; DiAntonio et al., 1999). Immunohistochemistry analyses of postsynaptic receptor distributions did not reveal an effect of the $p p k 29$ mutation on the spatial distribution of GluRIIA and GluRIIB subunits in larval muscles (Fig. 6A). Furthermore, quantitative analysis of fluorescence intensity revealed no significant differences in levels of protein at the NMJ between genotypes for GluRIIA, GluRIIB, or GluRIIC ( $n=4-6$ samples/group; ANOVA: GluRIIA, $F_{(1,7)}=$ $0.129, p=0.7298$; GluRIIB, $F_{(1,7)}=2.023, p=0.1979$; GluRIIC, $F_{(1,9)}=0.308, p=0.5923$; Fig. $\left.6 B\right)$. Quantitative real-time PCR 
(qRT-PCR) analyses, on the other hand, revealed significant shifts in the mRNA expression levels of GluRIIA and GluRIIB, but not in the common subunit GluRIIC, between wild-type and $p p k 29$ mutant larvae ( $n=4$ samples/group; ANOVA: GluRIIA, $F_{(1,6)}=6.981, p=0.0384 ;$ GluRIIB, $F_{(1,6)}=$ $15.414, p=0.0077$; GluRIIC, $F_{(1,6)}=0.057$, $p=0.8186$; Fig. $6 C$ ). Based on the known conductance properties of the postsynaptic GluRIIA and GluRIIB channels, these differences would not explain the observed phenotype but, instead, are more likely to represent transcriptional compensation for an overall reduction in GluRII receptor activity in the mutant muscle.

To further explore the impact of $p p k 29$ mutation on GluRII receptor activity, we conducted TEVC recordings of mini-excitatory junctional currents (mEJCs). Compared with wild-type animals, ppk29 mutants displayed decreased mEJC amplitude $\left(n=8-11 /\right.$ group; ANOVA: $F_{(1,17)}=$ 149.846, $p<0.0001)$ and current flow (charge; ANOVA: $F_{(1,17)}=51.823$, $p<0.0001)$, yet no change in the time constant of decay tau (ANOVA: $F_{(1,17)}=$ $0.789, p=0.3869$; Fig. 7). Together, these data suggest that $p p k 29$ mutant larvae display decreased current flow through GluRII receptors when compared with wild type, despite equal levels of GluRII receptors present at the NMJ.

\section{Discussion}

Despite their emerging importance in neurological and cognitive pathologies (Wemmie et al., 2013; Kreple et al., 2014), the precise neurophysiological functions of $\mathrm{DEG} / \mathrm{ENaC}$ channels remain elusive. Here we demonstrate that a Drosophila DEG/ENaCencoding gene, $p p k 29$, is required for normal synaptic functions. However, in contrast to the $p p k 11 / p p k 16$ complex (Younger et al., 2013), ppk29 action is restricted to the postsynaptic site and is associated with baseline spontaneous neurotransmission but not PhTX-dependent synaptic homeostasis. Therefore, we propose that individual $\mathrm{DEG} / \mathrm{ENaC}$-like channels may play independent roles in regulating synaptic functions, which may explain some of the contradicting reports about their functions in the mammalian synapse.

Previous studies have shown that some DEG/ENaC-encoding genes are expressed in human skeletal muscles (Gitterman et al., 2005), with their function remaining unknown. In Caenorhabditis elegans, the DEG/ENaC-encoding gene unc-105 is also expressed in muscles, where it is important for proper muscle organization, growth, and contraction (Liu et al., 1996; GarcíaAñoveros et al., 1998). Our data indicate that, in addition to the contributions of $\mathrm{DEG} / \mathrm{ENaC}$ proteins to muscle development and physiology, they also contribute to excitatory neurotransmission via postsynaptic processes in muscle. Nonetheless, because the fly NMJ is glutamatergic (Menon et al., 2013), the findings presented here could also provide important insights about postsynaptic functions of $\mathrm{DEG} / \mathrm{ENaC}$ signaling in glutamatergic central synapses of vertebrates.

The postsynaptic impact of $p p k 29$ on excitatory signaling may be mediated directly by PPK 29 or indirectly via interaction with other proteins. Since currently available tools did not allow us to localize PPK29 to specific subcellular compartments, it is too early to conclude whether the observed phenotypes in the ppk29 mutants are the consequence of a direct synaptic function or possibly of an indirect function via its action in other subcellular domains. We also note the possibility that PPK29 may play more than one role in muscles and, therefore, that the electrophysiological and behavioral data may not be mediated through the same mechanism.

Nevertheless, one intriguing way that PPK29 might directly contribute to synaptic transmission is by acting as a direct postsynaptic receptor for glutamate or other molecules that are coreleased during spontaneous excitatory neurotransmission. For example, in mouse brain slices, extracellular protons increase with the stimulation of glutamatergic inputs, which can activate acid-sensitive ion channels, which are also members of the DEG/ $\mathrm{ENaC}$ family (Du et al., 2014). Therefore, although we do not know yet whether PPK29 is an acid-activated channel, the corelease of protons with glutamate during spontaneous neurotransmission at the Drosophila NMJ may directly activate PPK29 channels.

The ppk29 gene may also affect neurotransmission indirectly through the modulation of expression or function of other synaptic proteins. Postsynaptic glutamate receptors are promising candidates to mediate an indirect impact of $p p k 29$ on synaptic physiology since these ionotropic receptors are the main mediators of excitatory neurotransmission at the larval NMJ (Schuster et al., 1991; Petersen et al., 1997; DiAntonio et al., 1999; Collins and DiAntonio, 2007). We found that ppk29 mutant animals exhibit decreased spontaneous amplitude and current flow, suggesting altered function of the postsynaptic ionotropic GluRs, which may be mediated by direct interaction between PPK29 and the GluR complex, or by indirect interaction via other postsynaptic signaling mechanisms. In line with this hypothesis, direct 
physical interactions between other DEG/ENaC proteins and potassium channels and sodium/chloride cotransporters have been reported in mammalian systems (Petroff et al., 2008; Mistry et al., 2016). We further hypothesize that the observed differences in GluR expression levels are due to compensatory transcriptional changes. We do not know whether the ppk29 mutation independently impacts both GluRIIA and GluRIIB. Yet, previous studies have shown that genetic manipulation of expression levels of either GluRIIA or GluRIIB affect expression levels of the other (Marrus et al., 2004); therefore, the ppk29 mutation may directly or indirectly impact one or both subunit types.

To date, studies of spontaneous neurotransmitter release at the Drosophila larval NMJ have suggested that its main function is to regulate the development and maintenance of excitatory synaptic transmission by regulating presynaptic morphology (Huntwork and Littleton, 2007; Choi et al., 2014) and the postsynaptic clustering of glutamate receptors (Saitoe et al., 2001). However, spontaneous neurotransmitter release at central synapses has also been shown to impact local protein synthesis in dendrites (Sutton et al., 2007) as well as dendritic summation of EPSPs (Sharma and Vijayaraghavan, 2003) at much shorter timescales. Here we have identified an important function for DEG/ENaC channels at the physiological timescale, which has an impact on both neurophysiological and behavioral phenotypes. Although we do not know yet how the molecular action of PPK29 might affect synapses and behavior, we argue that our findings about the contribution of $\mathrm{DEG} / \mathrm{ENaC}$-encoding genes to spontaneous excitatory neurotransmission at the Drosophila larval NMJ may serve as an excellent model for understanding the function of spontaneous baseline excitatory neurotransmission in regulating synaptic organization. Better understanding of these processes at the physiological timescale is essential for understanding behavioral and neural plasticity in health and disease.

\section{References}

An MC, Lin W, Yang J, Dominguez B, Padgett D, Sugiura Y, Aryal P, Gould TW, Oppenheim RW, Hester ME, Kaspar BK, Ko CP, Lee KF (2010) Acetylcholine negatively regulates development of the neuromuscular junction through distinct cellular mechanisms. Proc Natl Acad Sci U S A 107:10702-10707. CrossRef Medline

Ben-Shahar Y (2011) Sensory functions for degenerin/epithelial sodium channels (DEG/ENaC). Adv Genet 76:1-26. CrossRef Medline

Brand AH, Perrimon N (1993) Targeted gene expression as a means of altering cell fates and generating dominant phenotypes. Development 118: 401-415. Medline

Budnik V, Koh YH, Guan B, Hartmann B, Hough C, Woods D, Gorczyca M (1996) Regulation of synapse structure and function by the Drosophila tumor suppressor gene dlg. Neuron 17:627-640. CrossRef Medline

Cameron P, Hiroi M, Ngai J, Scott K (2010) The molecular basis for water taste in Drosophila. Nature 465:91-95. CrossRef Medline

Chen Y, Bharill S, Isacoff EY, Chalfie M (2015) Subunit composition of a DEG/ENaC mechanosensory channel of Caenorhabditis elegans. Proc Natl Acad Sci U S A 112:11690-11695. CrossRef Medline

Chen Z, Wang Q, Wang Z (2010) The amiloride-sensitive epithelial $\mathrm{Na}+$ channel PPK28 is essential for Drosophila gustatory water reception. J Neurosci 30:6247-6252. CrossRef Medline

Choi BJ, Imlach WL, Jiao W, Wolfram V, Wu Y, Grbic M, Cela C, Baines RA, Nitabach MN, McCabe BD (2014) Miniature neurotransmission regulates Drosophila synaptic structural maturation. Neuron 82:618-634. CrossRef Medline

Collins CA, DiAntonio A (2007) Synaptic development: insights from Drosophila. Curr Opin Neurobiol 17:35-42. CrossRef Medline

Davis GW (2013) Homeostatic signaling and the stabilization of neural function. Neuron 80:718-728. CrossRef Medline

DiAntonio A, Petersen SA, Heckmann M, Goodman CS (1999) Glutamate receptor expression regulates quantal size and quantal content at the Drosophila neuromuscular junction. J Neurosci 19:3023-3032. Medline
Dickman DK, Tong A, Davis GW (2012) Snapin is critical for presynaptic homeostatic plasticity. J Neurosci 32:8716-8724. CrossRef Medline

Du J, Reznikov LR, Price MP, Zha XM, Lu Y, Moninger TO, Wemmie JA, Welsh MJ (2014) Protons are a neurotransmitter that regulates synaptic plasticity in the lateral amygdala. Proc Natl Acad Sci U S A 111:89618966. CrossRef Medline

Eastwood AL, Goodman MB (2012) Insight into DEG/ENaC channel gating from genetics and structure. Physiology (Bethesda) 27:282-290. CrossRef

Frank CA, Kennedy MJ, Goold CP, Marek KW, Davis GW (2006) Mechanisms underlying the rapid induction and sustained expression of synaptic homeostasis. Neuron 52:663-677. CrossRef Medline

García-Añoveros J, García JA, Liu JD, Corey DP (1998) The nematode degenerin UNC-105 forms ion channels that are activated by degenerationor hypercontraction-causing mutations. Neuron 20:1231-1241. CrossRef Medline

Gitterman DP, Wilson J, Randall AD (2005) Functional properties and pharmacological inhibition of ASIC channels in the human SJ-RH30 skeletal muscle cell line. J Physiol 562:759-769. CrossRef Medline

Gonzales EB, Kawate T, Gouaux E (2009) Pore architecture and ion sites in acid-sensing ion channels and P2X receptors. Nature 460:599-604. CrossRef Medline

Hanson MG, Landmesser LT (2004) Normal patterns of spontaneous activity are required for correct motor axon guidance and the expression of specific guidance molecules. Neuron 43:687-701. CrossRef Medline

Huntwork S, Littleton JT (2007) A complexin fusion clamp regulates spontaneous neurotransmitter release and synaptic growth. Nat Neurosci 10: 1235-1237. CrossRef Medline

Ievglevskyi O, Isaev D, Netsyk O, Romanov A, Fedoriuk M, Maximyuk O, Isaeva E, Akaike N, Krishtal O (2016) Acid-sensing ion channels regulate spontaneous inhibitory activity in the hippocampus: possible implications for epilepsy. Philos Trans R Soc Lond B Biol Sci 371:20150431. CrossRef Medline

Jasti J, Furukawa H, Gonzales EB, Gouaux E (2007) Structure of acidsensing ion channel 1 at 1.9 A resolution and low pH. Nature 449:316323. CrossRef Medline

Kellenberger S, Schild L (2002) Epithelial sodium channel/degenerin family of ion channels: a variety of functions for a shared structure. Physiol Rev 82:735-767. CrossRef Medline

Kim YJ, Bao H, Bonanno L, Zhang B, Serpe M (2012) Drosophila Neto is essential for clustering glutamate receptors at the neuromuscular junction. Genes Dev 26:974-987. CrossRef Medline

Kreple CJ, Lu Y, Taugher RJ, Schwager-Gutman AL, Du J, Stump M, Wang Y, Ghobbeh A, Fan R, Cosme CV, Sowers LP, Welsh MJ, Radley JJ, LaLumiere RT, Wemmie JA (2014) Acid-sensing ion channels contribute to synaptic transmission and inhibit cocaine-evoked plasticity. Nat Neurosci 17:1083-1091. CrossRef Medline

Lin DM, Goodman CS (1994) Ectopic and increased expression of Fasciclin II alters motoneuron growth cone guidance. Neuron 13:507-523. CrossRef Medline

Lin H, Mann KJ, Starostina E, Kinser RD, Pikielny CW (2005) A Drosophila $\mathrm{DEG} / \mathrm{ENaC}$ channel subunit is required for male response to female pheromones. Proc Natl Acad Sci U S A 102:12831-12836. CrossRef Medline

Liu J, Schrank B, Waterston RH (1996) Interaction between a putative mechanosensory membrane channel and a collagen. Science 273:361364. CrossRef Medline

Liu L, Leonard AS, Motto DG, Feller MA, Price MP, Johnson WA, Welsh MJ (2003) Contribution of Drosophila DEG/ENaC genes to salt taste. Neuron 39:133-146. CrossRef Medline

Liu T, Starostina E, Vijayan V, Pikielny CW (2012) Two Drosophila DEG/ $\mathrm{ENaC}$ channel subunits have distinct functions in gustatory neurons that activate male courtship. J Neurosci 32:11879-11889. CrossRef Medline

Lu B, LaMora A, Sun Y, Welsh MJ, Ben-Shahar Y (2012) ppk23-Dependent chemosensory functions contribute to courtship behavior in Drosophila melanogaster. PLoS Genet 8:e1002587. CrossRef Medline

Marrus SB, Portman SL, Allen MJ, Moffat KG, DiAntonio A (2004) Differential localization of glutamate receptor subunits at the Drosophila neuromuscular junction. J Neurosci 24:1406-1415. CrossRef Medline

Mast JD, De Moraes CM, Alborn HT, Lavis LD, Stern DL (2014) Evolved differences in larval social behavior mediated by novel pheromones. Elife 3:e04205. CrossRef Medline

Menon KP, Carrillo RA, Zinn K (2013) Development and plasticity of the 
Drosophila larval neuromuscular junction. Wiley Interdiscip Rev Dev Biol 2:647-670. CrossRef Medline

Miller-Fleming TW, Petersen SC, Manning L, Matthewman C, Gornet M, Beers A, Hori S, Mitani S, Bianchi L, Richmond J, Miller DM (2016) The $\mathrm{DEG} / \mathrm{ENaC}$ cation channel protein UNC-8 drives activity-dependent synapse removal in remodeling GABAergic neurons. Elife 5:e14599. CrossRef Medline

Mistry AC, Wynne BM, Yu L, Tomilin V, Yue Q, Zhou Y, Al-Khalili O, Mallick R, Cai H, Alli AA, Ko B, Mattheyses A, Bao HF, Pochynyuk O, Theilig F, Eaton DC, Hoover RS (2016) The sodium chloride cotransporter (NCC) and epithelial sodium channel (ENaC) associate. Biochem J 473:3237-3252. CrossRef Medline

Petersen SA, Fetter RD, Noordermeer JN, Goodman CS, DiAntonio A (1997) Genetic analysis of glutamate receptors in Drosophila reveals a retrograde signal regulating presynaptic transmitter release. Neuron 19: 1237-1248. CrossRef Medline

Petroff EY, Price MP, Snitsarev V, Gong H, Korovkina V, Abboud FM, Welsh MJ (2008) Acid-sensing ion channels interact with and inhibit BK K+ channels. Proc Natl Acad Sci U S A 105:3140-3144. CrossRef Medline

Saitoe M, Schwarz TL, Umbach JA, Gundersen CB, Kidokoro Y (2001) Absence of junctional glutamate receptor clusters in Drosophila mutants lacking spontaneous transmitter release. Science 293:514-517. CrossRef Medline

Schindelin J, Arganda-Carreras I, Frise E, Kaynig V, Longair M, Pietzsch T, Preibisch S, Rueden C, Saalfeld S, Schmid B, Tinevez JY, White DJ, Hartenstein V, Eliceiri K, Tomancak P, Cardona A (2012) Fiji: an opensource platform for biological-image analysis. Nat Methods 9:676-682. CrossRef Medline

Schuster CM, Ultsch A, Schloss P, Cox JA, Schmitt B, Betz H (1991) Molecular cloning of an invertebrate glutamate receptor subunit expressed in Drosophila muscle. Science 254:112-114. CrossRef Medline

Sharma G, Vijayaraghavan S (2003) Modulation of presynaptic store calcium induces release of glutamate and postsynaptic firing. Neuron 38 : 929-939. CrossRef Medline

Starostina E, Liu T, Vijayan V, Zheng Z, Siwicki KK, Pikielny CW (2012) A Drosophila DEG/ENaC subunit functions specifically in gustatory neurons required for male courtship behavior. J Neurosci 32:4665-4674. CrossRef Medline
Sutton MA, Taylor AM, Ito HT, Pham A, Schuman EM (2007) Postsynaptic decoding of neural activity: eEF2 as a biochemical sensor coupling miniature synaptic transmission to local protein synthesis. Neuron 55:648661. CrossRef Medline

Thistle R, Cameron P, Ghorayshi A, Dennison L, Scott K (2012) Contact chemoreceptors mediate male-male repulsion and male-female attraction during drosophila courtship. Cell 149:1140-1151. CrossRef Medline

Urbano FJ, Lino NG, González-Inchauspe CM, González LE, Colettis N, Vattino LG, Wunsch AM, Wemmie JA, Uchitel OD (2014) Acid-sensing ion channels 1a (ASICla) inhibit neuromuscular transmission in female mice. Am J Physiol Cell Physiol 306:C396-C406. CrossRef Medline

Vijayan V, Thistle R, Liu T, Starostina E, Pikielny CW (2014) Drosophila pheromone-sensing neurons expressing the ppk25 ion channel subunit stimulate male courtship and female receptivity. PLoS Genet 10: e1004238. CrossRef Medline

Voglis G, Tavernarakis N (2008) A synaptic DEG/ENaC ion channel mediates learning in $\mathrm{C}$. elegans by facilitating dopamine signalling. EMBO J 27:3288-3299. CrossRef Medline

Wemmie JA, Chen J, Askwith CC, Hruska-Hageman AM, Price MP, Nolan BC, Yoder PG, Lamani E, Hoshi T, Freeman JH Jr, Welsh MJ (2002) The acid-activated ion channel ASIC contributes to synaptic plasticity, learning, and memory. Neuron 34:463-477. CrossRef Medline

Wemmie JA, Taugher RJ, Kreple CJ (2013) Acid-sensing ion channels in pain and disease. Nat Rev Neurosci 14:461-471. CrossRef Medline

Younger MA, Müller M, Tong A, Pym EC, Davis GW (2013) A presynaptic $\mathrm{ENaC}$ channel drives homeostatic plasticity. Neuron 79:1183-1196. CrossRef Medline

Yuan Q, Song Y, Yang CH, Jan LY, Jan YN (2014) Female contact modulates male aggression via a sexually dimorphic GABAergic circuit in Drosophila. Nat Neurosci 17:81-88. CrossRef Medline

Zelle KM, Lu B, Pyfrom SC, Ben-Shahar Y (2013) The genetic architecture of degenerin/epithelial sodium channels in Drosophila. G3 (Bethesda) 3:441-450. CrossRef Medline

Zheng X, Valakh V, Diantonio A, Ben-Shahar Y (2014) Natural antisense transcripts regulate the neuronal stress response and excitability. Elife 3:e01849. CrossRef Medline 